\title{
Optimal system, nonlinear self-adjointness and conservation laws for generalized shallow water wave equation
}

https://doi.org/10.1515/phys-2018-0049

Received August 19, 2017; accepted January 9, 2018

\begin{abstract}
In this article, the generalized shallow water wave (GSWW) equation is studied from the perspective of one dimensional optimal systems and their conservation laws (Cls). Some reduction and a new exact solution are obtained from known solutions to one dimensional optimal systems. Some of the solutions obtained involve expressions with Bessel function and Airy function [1-3]. The GSWW is a nonlinear self-adjoint (NSA) with the suitable differential substitution, Cls are constructed using the new conservation theorem.
\end{abstract}

Keywords: GSWW,optimal system, Cls, infinitesimal generators, NSA

PACS: 02.20.Qs; 02.20.Sv; 02.30.Hq; 02.30.Jr

\section{Introduction}

Lie symmetry methods play a vital role in the study and finding solutions for nonlinear partial differential equations (NLPDE) [4-16]. Different techniques are used in the literature for the construction of Cls for different system of equation and these Cls are important for the investigation of a physical system [4-21]. Moreover, Authors made rigorous attempts for construction of the construction of onedimensional and higher-dimensional optimal systems optimal system of Lie algebra [22-26]. Cls and symmetries have many application in science, physics and engineering [32-37].

\footnotetext{
`Corresponding Author: Dumitru Baleanu: Cankaya University, Department of Mathematics, Ögretmenler Cad. 1406530, Ankara, Turkey / Institute of Space Sciences, Magurele, Bucharest, Romania, E-mail: dumitru@cankaya.edu.tr Mustafa Inc, Abdullahi Yusuf, Aliyu Isa Aliyu: Firat University, Science Faculty, Department of Mathematics, 23119, Elazig, Turkey, E-mail: minc@firat.edu.tr, yusufabdullahi@fud.edu.ng, aliyu.isa@fud.edu.ng
}

In this work, we obtain one-dimensional optimal system, exact solutions and Cls for the GSWW equation given by

$$
\Delta=u_{x x x t}+a u_{x} u_{x t}+b u_{t} u_{x x}-u_{x t}-u_{x x}=0,
$$

where $a \neq 0, b \neq 0$ are arbitrary constants. Eq. (1) have been studied by different authors using a variety of techniques. For example [27] introduced exact solutions for Eq. (1) by the general projective Ricatti equations method. Periodic wave solution for Eq. (1) by the improved Jacobi elliptic function method was investigated by [28] . Homogeneous balance method [29] was applied to investigate some solutions for Eq. (1) and some new solution of Eq. (1) with extended elliptic function method was proposed in [30] and many more.

\section{One-dimensional optimal system of subalgebras of GSWW}

In this section, we establish the optimal system of onedimensional subalgebras of $L_{4}$ and their corresponding exact solutions. Consider one parameter Lie group of the infinitesimal transformation below

$$
\begin{aligned}
& \bar{x}=x+\epsilon \xi^{1}(x, t, u)+O\left(\epsilon^{2}\right), \\
& \bar{t}=t+\epsilon \xi^{2}(x, t, u)+O\left(\epsilon^{2}\right), \\
& \bar{u}=u+\epsilon \eta(x, t, u)+O\left(\epsilon^{2}\right),
\end{aligned}
$$

where $\epsilon$ is the group parameter. The corresponding Lie algebra of the infinitesimal symmetries is the set of vector field of the form

$$
X=\xi^{1}(x, t, u) \frac{\partial}{\partial t}+\xi^{2}(x, t, u) \frac{\partial}{\partial x}+\eta(x, t, u) \frac{\partial}{\partial u} .
$$

Considering the fourth order prolongation $\operatorname{Pr}^{(4)}$ of the vector field $X$ such that

$$
\operatorname{Pr}^{(4)} X(\Delta)=0,
$$


where $\Delta=(1)$, whenever $\Delta=0$. Using SYM package introduced in [31], the determining equations for Eq. (1) are obtained. Solving for $\eta(x, t, u), \xi^{2}(x, t, u)$, and $\xi^{1}(x, t, u)$ from the obtained determining equations, we get

$$
\begin{aligned}
\xi^{1} & =c_{1}+\frac{1}{2} a x c_{3}, \\
\xi^{2} & =-\frac{1}{2} a t c_{3}+c_{4}+b F(t), \\
\eta & =c_{2}-\frac{1}{2} a u c_{3}+x c_{3}+F(t) .
\end{aligned}
$$

where $c_{1}, c_{2}, c_{3}$ and $c_{4}$ are arbitrary constants and $F(t)$ is an arbitrary function of $t$. The Lie symmetry algebra admitted by Eq. (1) is spanned by four infinitesimals generators below

$$
\begin{aligned}
& X_{1}=\partial_{x}, \\
& X_{2}=\partial_{u}, \\
& X_{3}=\partial_{t}, \\
& X_{4}=-a t \partial_{t}-(a u-2 x) \partial_{u}+a x \partial_{x} .
\end{aligned}
$$

The corresponding commutator table of the infinitesimal generators is given by

Table 1: Commutator table of the Lie algebra for GSWWE

\begin{tabular}{l|llll}
\hline$\left[X_{i}, X_{j}\right]$ & $X_{1}$ & $X_{2}$ & $X_{3}$ & $X_{4}$ \\
\hline$X_{1}$ & 0 & 0 & 0 & $a X_{1}+2 X_{2}$ \\
$X_{2}$ & 0 & 0 & 0 & $-a X_{2}$ \\
$X_{3}$ & 0 & 0 & 0 & $-a X_{3}$ \\
$X_{4}$ & $-a X_{1}-2 X_{2}$ & $a X_{2}$ & $a X_{3}$ & 0 \\
\hline
\end{tabular}

\subsection{Construction of one-dimensional optimal system of subalgebras}

The Lie algebra $L_{4}$ spanned by the given generators $X_{1}$, $X_{2}, X_{3}$, and $X_{4}$ can guarantee a possibility to obtain invariant solutions of Eq. (1). This will be based mainly on one-dimensional subalgebra of $L_{4}$. There may be an infinite number of one-dimensional subalgebras of $L_{4}$. Therefore, one can write an arbitrary generators from $L_{4}$ as

$$
X=l^{1} X_{1}+l^{2} X_{2}+l^{3} X_{3}+l^{4} X_{4},
$$

which depend on the four arbitrary constants $l^{1}, l^{2}, l^{3}$, and $l^{4}$. We construct the one-dimensional optimal system of subalgebras using the method introduced in [22-26]. After the transformation of $L_{4}$, we can get a 4-parameter group of linear transformations of the generators as

$$
l=\left(l^{1}, l^{2}, l^{3}, l^{4}\right) .
$$

where $l^{1}, l^{2}, l^{3}$, and $l^{4}$ are the coefficients in Eq. (13).

\subsection{Linear transformation}

Here, we investigate the linear transformations by using their generators which is given as

$$
E_{\mu}=c_{\mu \nu}^{\lambda} l^{v} \frac{\partial}{\partial l^{\lambda}}, \quad \mu=1, \ldots, 4 .
$$

and the structure constants of the Lie algebra $L_{4}$ defined by $c_{\mu \nu}^{\lambda}$ is given as

$$
\left[X_{\mu}, X_{v}\right]=c_{\mu \nu}^{\lambda} X_{\lambda}
$$

Consider the following cases:

- Case 1: For $\mu=1, v=4$, and $\lambda=1,2$ in Table 1. $\left[X_{1}, X_{4}\right]=c_{14}^{1} X_{1}+c_{14}^{2} X_{2}$ and the non vanishing structure constants are $\left(c_{\mu \nu}^{\lambda}\right)$ are $c_{14}^{1}=a, c_{14}^{2}=2$.

- Case 2: For $\mu=2, v=4$, and $\lambda=2$ in Table 1. $\left[X_{2}, X_{4}\right]=$ $c_{24}^{2} X_{2}$ and the non vanishing structure constants $\left(c_{\mu \nu}^{\lambda}\right)$ are $c_{24}^{2}=-a$.

- Case 3: For $\mu=3, v=4$, and $\lambda=3$ in Table 1. $\left[X_{3}, X_{4}\right]=$ $c_{34}^{3} X_{3}$ and the non vanishing structure constants $\left(c_{\mu \nu}^{\lambda}\right)$ are $c_{34}^{3}=-a$.

- Case 4: For $\mu=4, v=1$, and $\lambda=1,2$ in Table 1. $\left[X_{4}, X_{1}\right]=c_{41}^{1} X_{1}+c_{41}^{2} X_{2}$ and the non vanishing structure constants $\left(c_{\mu \nu}^{\lambda}\right)$ are $c_{41}^{1}=-a, c_{41}^{2}=-2$. Setting $v=$ $2, \lambda=2$ row four column two, we get $\left[X_{4}, X_{2}\right]=c_{52}^{2} X_{2}$ and $c_{42}^{2}=a$, Setting $v=3, \lambda=3$ row four column three, we get $\left[X_{4}, X_{3}\right]=c_{43}^{3} X_{3}$ and $c_{43}^{3}=a$.

Now, Substituting the values of the non-vanishing structure constants in Eq. (15) for $\mu=1,2, \ldots, 4$, we obtain

$$
\begin{array}{r}
E_{1}=a l^{4} \frac{\partial}{\partial l^{1}}+2 l^{4} \frac{\partial}{\partial l^{2}}, \\
E_{2}=-a l^{4} \frac{\partial}{\partial l^{2}}, \\
E_{3}=-a l^{4} \frac{\partial}{\partial l^{3}}, \\
E_{4}=-a l^{1} \frac{\partial}{\partial l^{1}}-2 l^{1} \frac{\partial}{\partial l^{2}}+a l^{2} \frac{\partial}{\partial l^{2}}+a l^{3} \frac{\partial}{\partial l^{3}} .
\end{array}
$$




\subsection{Lie equation}

To obtain the Lie equation, we integrate the generators $E_{1}$, $E_{2}, E_{3}, E_{4}$ in Eq. (17) using the initial condition $\left.\bar{l}\right|_{\epsilon=0}=l$.

- For the generator $E_{1}$, the Lie equation with the parameter $\epsilon$ are given by

$\frac{\partial \overline{1}}{\partial \epsilon}=a \overline{l^{4}}, \frac{\partial \overline{l^{2}}}{\partial \epsilon}=2 \overline{l^{4}}, \frac{\partial \bar{l}}{\partial \epsilon}=0, \frac{\partial \overline{l^{4}}}{\partial \epsilon}=0$. Integrating and using the initial condition we obtain $\overline{l^{1}}=a \epsilon_{1} l^{4}+l^{1}$, $\overline{l^{2}}=2 \epsilon_{1} l^{4}+l^{2}, \overline{l^{3}}=l^{3}, \overline{l^{4}}=l^{4}$.

similarly for the other generators by following the same approach we get

- For $E_{2}$, we obtain $\overline{l^{1}}=l^{1}, \overline{l^{2}}=-a \epsilon_{2} l^{4}+l^{2}, \overline{l^{3}}=l^{3}$, $\overline{l^{4}}=l^{4}$.

- For $E_{3}$, we have $\overline{l^{1}}=l^{1}, \overline{l^{2}}=l^{2}, \overline{l^{3}}=-a \epsilon_{3} l^{4}+l^{3}$ and $\overline{l^{4}}=$ $l^{4}$.

- For $E_{4}$, we have $\overline{l^{1}}=\frac{l^{1}}{1+a \epsilon_{4}}, \overline{l^{2}}=\frac{-2 \epsilon_{1}+l^{2}}{1-a \epsilon_{4}}, \overline{l^{3}}=\frac{l^{3}}{1-a \epsilon_{4}}$ and $\overline{l^{4}}=l^{4}$.

using SYM package [31], we can get the optimal system raw data in matrix form as:

$$
\left(\begin{array}{cccc}
a \epsilon_{1} l^{4}+l^{1} & 2 \epsilon_{1} l^{4}+l^{2} & l^{3} & l^{4} \\
l^{1} & -a \epsilon_{2} l^{4}+l^{2} & l^{3} & l^{4} \\
l^{1} & l^{2} & -a \epsilon_{3} l^{4}+l^{3} & l^{4} \\
\frac{l^{1}}{1+a \epsilon_{4}} & \frac{-2 \epsilon_{1}+l^{2}}{1-a \epsilon_{4}} & \frac{l^{3}}{1-a \epsilon_{4}} & l^{4}
\end{array}\right)
$$

and the number of the functionally invariants, which is found to be $l^{4}$. The corresponding one-dimensional optimal system of subalgebras are found to be the following:
1. $X_{3}$,
2. $\alpha X_{2}+X_{3}$,
3. $X_{4}$,
4. $X_{1}+X_{4}$,
5. $\alpha X_{3}+X_{4}$,
6. $\alpha X_{1}+\beta X_{2}+X_{3}$,

where $\alpha, \beta \in \mathbb{R}$. In the following, we list the corresponding similarity variables, similarity solutions as well as the reduced PDEs obtained from the generators of optimal system and their exact solutions.

1. Similarity variable related to $X_{3}$ is $u(x, t)=F(x)$ and $F(x)$ satisfies $F_{X x}=0$ two times integration implies that $F(x)=c_{1}+x c_{2}$ and we have the exact solution

$$
u(x, t)=c_{1}+x c_{2} .
$$

2. Similarity variable related to $\alpha X_{2}+X_{3}$ is $u(x, t)=\alpha t+$ $F(x)$ and $F(x)$ satisfies $F_{x x}=0$ which after integrating twice gives $F(x)=c_{1}+x c_{2}$ and we have the exact so- lution

$$
u(x, t)=\alpha t+c_{1}+x c_{2} .
$$

3. Similarity variable related to $X_{4}$ is $u(x, t)=\frac{x^{2}+a F(\zeta)}{a x}$, $\zeta=t x$ and $F(\zeta)$ satisfies

$$
\begin{array}{r}
F(\zeta)\left(2+2 b F_{\zeta}-a \zeta F_{\zeta \zeta}+\zeta\left[-2 b F_{\zeta}^{2}\right.\right. \\
+F_{\zeta}\left(-2+(a+b) \zeta F_{\zeta \zeta}\right)+\zeta\left(F_{\zeta \zeta}\right. \\
\left.\left.+\zeta F_{\zeta \zeta \zeta \zeta}\right)\right]=0,
\end{array}
$$

thrice integration of Eq. (20) and letting $c_{1}=0$ yields

$$
4 \zeta F_{\zeta}+\frac{1}{4} \zeta^{2}\left((a+b) F^{2}(\zeta)-36 F(\zeta)\right)+\left(c_{3} \zeta+c_{2}\right) \zeta=0,
$$

solving for $F(\zeta)$ in Eq. (21) we obtain

$F(\zeta)=\frac{2\left\{9 \operatorname{BesselJ}\left(9, \frac{\sqrt{-a-b} \sqrt{-c_{1} \zeta-c_{2}}}{\sqrt{\zeta}}\right) c_{4}+\frac{Q_{0} Q_{1}-Q_{2}+Q_{3}}{2 \sqrt{\zeta}}\right\}}{L_{1}-L_{2}}$,

where

$$
\begin{gathered}
L_{1}=-(a+b) \operatorname{BesselJ}\left(9, \frac{\sqrt{-a-b} \sqrt{-c_{1} \zeta-c_{2}}}{\sqrt{\zeta}}\right), \\
\left.L_{2}=(a+b) \operatorname{BesselJ}\left(9, \frac{\sqrt{-a-b} \sqrt{-c_{1} \zeta-c_{2}}}{\sqrt{\zeta}}\right) c_{4}\right), \\
Q_{0}=\sqrt{-a-b} \sqrt{-c_{1} \zeta-c_{2}}, \\
Q_{1}=-2 \operatorname{Bessel}\left(10, \frac{\sqrt{-a-b} \sqrt{-c_{1} \zeta-c_{2}}}{\sqrt{x}}\right), \\
Q_{2}=\operatorname{BesselJ}\left(8, \frac{\sqrt{-a-b} \sqrt{-c_{1} \zeta-c_{2}}}{\sqrt{\zeta}}\right) c_{1}, \\
Q_{3}=\operatorname{BesselJ}\left(10, \frac{\sqrt{-a-b} \sqrt{-c_{1} \zeta-c_{2}}}{\sqrt{\zeta}}\right) c_{4} .
\end{gathered}
$$

Hence by back substituting the similarity variables we get the exact solution as

$$
u(x, t)=\frac{x}{a}+E_{0},
$$

where

$E_{0}=\frac{2\left\{9 \operatorname{BesselJ}\left(9, \frac{\left.\sqrt{-a-b} \sqrt{-c_{1} \zeta-c_{2}}\right)}{\sqrt{\zeta}} c_{4}+\frac{Q_{0} Q_{1}-Q_{2}+Q_{3}}{2 \sqrt{\zeta}}\right\}\right.}{x\left(L_{1}-L_{2}\right)}$,

$L_{1}, L_{2}$ are as stated above and $\zeta=t x$. 
4. Similarity variable related to $X_{1}+X_{4}$ is $u(x, t)=\frac{x^{2}+F(\zeta)}{1+a x}$, $\zeta=t(1+a x)$ and $F(\zeta)$ satisfies the following

$$
\begin{array}{r}
2-2 a^{2} b \zeta F_{\zeta}^{2}-a \zeta F_{\zeta \zeta}+a^{2} \zeta^{2} F_{\zeta \zeta} \\
+a^{2} F(\zeta)\left(2+2 b F_{\zeta}-a \zeta F_{\zeta \zeta}\right)+F_{x}\left(2 b-2 a^{2} \zeta\right. \\
\left.+a^{2}(a+b) \zeta^{2} F_{\zeta \zeta}\right)+a^{3} \zeta^{3} F_{\zeta \zeta \zeta}=0,
\end{array}
$$

three times integration and letting $c_{1}=c_{2}=c_{3}=0$ in the above equation, gives

$$
\left(4 a \zeta F_{\zeta}+(a+b) F^{2}(\zeta)-36 a F(\zeta)\right) \frac{1}{4} a^{2} \zeta^{2}+\frac{\zeta^{3}}{3}=0,
$$

solving for $F(\zeta)$ we have the following equation in Bessel function form

$$
F(\zeta)=-\frac{4 a \zeta\left(P_{0}+G_{0}+G_{2}+G_{3}\right)}{G_{6}},
$$

where

$$
\begin{gathered}
P_{0}=\frac{-\left(a \alpha^{2}-b \alpha^{2}\right)^{\frac{9}{2}} \zeta^{\frac{7}{2}} \operatorname{Bessel}\left[9, \frac{\sqrt{-a \alpha^{2}-b \alpha^{2}} \sqrt{\zeta}}{a^{2} \sqrt{3}}\right]}{4608 a^{18} \sqrt{3} a^{18}}, \\
G_{0}=\frac{-\left(a \alpha^{2}-b \alpha^{2}\right)^{5} \zeta^{4}\left(B e s s e l K\left[8, \frac{\sqrt{-a \alpha^{2}-b \alpha^{2}} \sqrt{\zeta}}{a^{2} \sqrt{3}}\right]-G_{1}\right)}{248832 a^{20}}, \\
G_{2}=-c_{4} \frac{\sqrt{3}\left(-a \alpha^{2}-b \alpha^{2}\right)^{\frac{9}{2}} B e s s e l K\left[9, \frac{\sqrt{-a \alpha^{2}-b \alpha^{2}} \sqrt{\zeta}}{a^{2} \sqrt{3}}\right]}{8 a^{18}(a+b)^{\frac{9}{2}} \alpha^{9}}, \\
G_{3}=c_{4} \frac{35\left(-a \alpha^{2}-b \alpha^{2}\right)^{5} \zeta^{4} G_{4}}{48 a^{18}(a+b)^{\frac{9}{2}} \alpha^{9} a^{20}}, \\
G_{4}=\left(B e s s e l K\left[8, \frac{\sqrt{-a \alpha^{2}-b \alpha^{2}} \sqrt{\zeta}}{a^{2} \sqrt{3}}\right]+G_{5},\right. \\
G_{6}=\frac{\left(-a \alpha^{2}-b \alpha^{2}\right)^{\frac{9}{2}} B e s s e l K\left[9, \frac{\sqrt{-a \alpha^{2}-b \alpha^{2}} \sqrt{\zeta}}{a^{2} \sqrt{3}}\right]}{20736 a^{18} \sqrt{3}}-G_{7}, \\
G_{7}=\frac{\left.B \operatorname{Bessel} K\left[10, \frac{\sqrt{-a \alpha^{2}-b \alpha^{2}} \sqrt{\zeta}}{a^{2} \sqrt{3}}\right]\right),}{35\left(-a \alpha^{2}-b \alpha^{2}\right)^{\frac{9}{2}} B e s s e l K\left[9, \frac{\sqrt{-a \alpha^{2}-b \alpha^{2}} \sqrt{\zeta}}{a^{2} \sqrt{3}}\right] c_{4}} .
\end{gathered}
$$

and the exact solution is

$$
u(x, t)=\frac{x^{2}}{1+a x}-\frac{4 a \zeta\left(P_{0}+G_{0}+G_{2}+G_{3}\right)}{G_{6}},
$$

where $\zeta=t(1+a x)$.
5. Similarity variable related to $\beta X_{3}+X_{4}$ is $u(x, t)=$ $\frac{x^{2}+a F(\zeta)}{a x}, \zeta=\frac{(a t-\alpha) x}{a}$ and $F(\zeta)$ satisfies the following

$$
\begin{array}{r}
F(\zeta)\left(2+2 b F_{\zeta}-a \zeta F_{\zeta \zeta}+\zeta\left[-2 b F_{\zeta}^{2}\right.\right. \\
+F_{\zeta}\left(-2+(a+b) \zeta F_{\zeta \zeta}\right)+\zeta\left(F_{\zeta \zeta}\right. \\
\left.+\zeta F_{\zeta \zeta \zeta \zeta}\right)=0,
\end{array}
$$

here the reduced PDE is the same as that in reduction 3 , the only different is the variable $\zeta$. Therefore, we get

$$
u(x, t)=\frac{x}{a}+E
$$

where

$$
E=\frac{2\left\{9 \operatorname{Bessel}\left(9, \frac{\sqrt{-a-b} \sqrt{-c_{1} \zeta-c_{2}}}{\sqrt{\zeta}}\right) c_{4}+\frac{Q_{0} Q_{1}-Q_{2}+Q_{3}}{2 \sqrt{\zeta}}\right\}}{x\left(L_{1}-L_{2}\right)}
$$

and $L_{1}, L_{2}, Q_{0}, Q_{1}, Q_{2}$, and $Q_{3}$ are as stated in Eq. (23) and $\zeta=\frac{(a t-\alpha) x}{a}$.

6. Similarity variable related to $\alpha X_{1}+\beta X_{2}+X_{3}$ is $u(x, t)=$ $\frac{\beta x+\alpha F(\zeta)}{\alpha}, \zeta=\frac{\alpha t-x}{\alpha}$ and $F(\zeta)$ satisfies the following

$$
\left.\alpha\left(1+\alpha-a \beta+(a+b) F_{\zeta}\right) F_{\zeta \zeta}-F_{\zeta \zeta \zeta \zeta}\right)=0,
$$

three times integration of Eq. (30) with $c_{1}=0$ leads

$$
F_{\zeta}-\frac{1}{4}(a+b) \alpha F^{2}(\zeta)+\left(c_{3}+\zeta c_{2}\right) \zeta=0
$$

solving for $F_{\zeta}$, we obtain

$$
F(\zeta)=\frac{4(a \alpha+b \alpha)}{2^{\frac{2}{3}}\left(c_{2}(a \alpha+b \alpha)\right)^{\frac{2}{3}}}\left[\frac{A_{1}+A_{2}}{(a \alpha+b \alpha)\left(A_{3}+A_{4}\right)}\right],
$$

where

$$
\begin{aligned}
& A_{1}=\operatorname{AiryBiPrime}\left[\frac{2^{\frac{4}{3}\left(\frac{1}{4} c_{3}(a \alpha+b \alpha)+\frac{1}{4} c_{2}(a \alpha+b \alpha) \zeta\right)}}{\left(c_{2}(a \alpha+b \alpha)^{\frac{2}{3}}\right)}\right], \\
& A_{2}=\operatorname{AiryAiPrime}\left[\frac{2^{\frac{4}{3}\left(\frac{1}{4} c_{3}(a \alpha+b \alpha)+\frac{1}{4} c_{2}(a \alpha+b \alpha) \zeta\right) c_{4}}}{\left(c_{2}(a \alpha+b \alpha)^{\frac{2}{3}}\right)}\right], \\
& A_{3}=\operatorname{AiryBiPrime}\left[\frac{2^{\frac{4}{3}\left(\frac{1}{4} c_{3}(a \alpha+b \alpha)+\frac{1}{4} c_{2}(a \alpha+b \alpha) \zeta\right)}}{\left(c_{2}(a \alpha+b \alpha)^{\frac{2}{3}}\right)}\right], \\
& A_{4}=\operatorname{AiryAiPrime}\left[\frac{2^{\frac{4}{3}\left(\frac{1}{4} c_{3}(a \alpha+b \alpha)+\frac{1}{4} c_{2}(a \alpha+b \alpha) \zeta\right) c_{4}}}{\left(c_{2}(a \alpha+b \alpha)^{\frac{2}{3}}\right)}\right],
\end{aligned}
$$

Thus, by back substituting the similarity variables we get

$u(x, t)=\frac{\beta x}{\alpha}+\frac{4(a \alpha+b \alpha)}{2^{\frac{2}{3}}\left(c_{2}(a \alpha+b \alpha)\right)^{\frac{2}{3}}}\left[\frac{A_{1}+A_{2}}{(a \alpha+b \alpha)\left(A_{3}+A_{4}\right)}\right]$

where $\zeta=\frac{\alpha t-x}{\alpha}$. 


\section{Physical interpretation of the solutions (23) and (33)}

In order to have clear and proper understanding of the physical properties of the power series solution, the 3-D, 2-D and contour plots for the solution Eqs. (23) and (33) are plotted in Figures 1-4 by using suitable parameter values.

\section{Nonlinear self-adjointness}

The system of $\bar{m}[20,21]$ differential equations

$$
F_{\bar{\alpha}}\left(x, u, u_{(1)}, \ldots, u_{(s)}\right)=0,
$$

$\bar{\alpha}=1, \ldots, \bar{m}$, with $m$ dependent variables $u=\left(u^{1}, \ldots, u^{m}\right)$ is said to be NSA if the adjoint equations

$$
F_{\alpha}^{\star}\left(x, u, u_{(1)}, v_{(1)} \ldots, u_{(s)}, v_{(s)}\right) \equiv \frac{\delta\left(v^{\bar{\beta}} F_{\bar{\beta}}\right)}{\delta u^{\alpha}}=0,
$$

$\alpha=1, \ldots, m$, are satisfied for all solutions $u$ of the original system Eq. (34) upon a substitution

$$
v^{\bar{\alpha}}=\varphi^{\bar{\alpha}}(x, u),
$$

$\bar{\alpha}=1, \ldots, \bar{m}$, such that

$$
\varphi(x, u) \neq 0 .
$$

On the other hand, the equation below holds:

$F_{\alpha}^{\star}\left(x, u, \varphi(x, u), \ldots, u_{(s)}, \varphi_{(s)}\right)=\lambda_{\alpha}^{\bar{\beta}} F_{\bar{\beta}}\left(x, u, \ldots, u_{(s)}\right)$,

$\alpha=1, \ldots, m$, where $\lambda_{\alpha}^{\bar{\beta}}$ are undetermined coefficients, and $\varphi(\sigma)$ are derivatives of Eq. (36),

$$
\varphi(\sigma)=\left\{D_{i_{1}} \ldots D_{i_{\sigma}}\left(\varphi^{\bar{\alpha}}(x, u)\right)\right\}
$$

, $\sigma=1, \ldots, s$. Here $v$ and $\varphi$ are the $\bar{m}$-dimensional vectors $v=\left(v^{1}, \ldots, v^{(m)}\right), \varphi=\left(\varphi^{1}, \ldots, \varphi^{\bar{m}}\right)$, and also, it is worth noting that not all components $\varphi^{\bar{\alpha}}(x, u)$ of $\varphi$ vanish simultaneously from Eq. (37).

\subsection{Test for self-adjointness for GSWW}

Here, we want to test the self-adjointness of Eq. (1). The adjoint equation for Eq. (1) is given by

$$
\begin{gathered}
F^{\star}=2 b v_{x} u_{x t}+\left(-1+a u_{x}\right) v_{x t}+a v_{t} u_{x x} \\
-b v_{t} u_{x x}+\left(-1+b u_{t}\right) v_{x x}+v_{x x x t}=0 .
\end{gathered}
$$

Let $v=\phi(x, t, u)$, after some calculations and equating the coefficients of the derivatives $u_{t}, u_{x}, u_{x t}, u_{x x}, u_{x x t}$ and $u_{x x x}$ to zero, we have

$$
\begin{gathered}
\phi_{u u}=0 \\
3 \phi_{, u u}=0 \\
3 \phi_{, u u u}=0, \\
\phi_{\text {uиuu }}=0, \\
\phi_{t u}=0, \\
3 \phi_{t u u}=0, \\
\phi_{t u u u}=0, \\
3 \phi_{x u}=0, \\
(a-b) \phi_{u}+3 \phi_{x u u}=0, \\
2\left(b \phi_{u}+3 \phi_{x u u}\right)=0, \\
(a+b) \phi_{u u}+3 \phi_{x u u u}=0, \\
a \phi_{t}-b \phi_{t}+3 \phi_{x t u}=0, \\
-\phi_{u u}+a \phi_{t u}+3 \phi_{x t u u}=0, \\
2 b \phi_{x}+3 \phi_{x x u}=0, \\
-\phi_{u u}+a \phi_{x u}+2 b \phi_{x u}+3 \phi_{x x u u}=0, \\
-\phi_{t u}-2 \phi_{x u}+a \phi_{x t}+3 \phi_{x x t u}=0, \\
-\phi_{x u}+b \phi_{x x}+\phi_{x x x u}=0, \\
-\phi_{x t}-\phi_{x x}+\phi_{x x x t}=0 .
\end{gathered}
$$

The solution for $\phi(t, x, u)$ from the above equation is simply found to be the following

$$
\phi(t, x, u)=C_{1} \text { for }(a-b) b \neq 0,
$$

where $C_{1}$ is an arbitrary constant. Therefore, Eq. (1) is NSA with the substitution in Eq. (40).

\section{Conservation laws for GSWW}

In this section, we establish Cls for Eq. (1) [19-21].

The reality that Eq. (1) is NSA with the obtained differential substitution in Eq. (40), we can use the Noether operator $\mathcal{N}$ to obtain its conserved vectors $\left(C^{1}, C^{2}\right)$ [17-20]. The obtained conserved vectors will satisfy the conservation equation $D_{x} C^{1}+D_{t} C^{2}=0$. Moreover, the non local variables appearing in that formula must be substituted according to equation (40). 


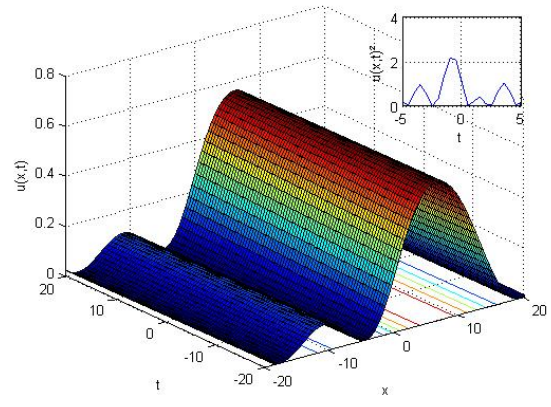

Figure 1: 3D plot of (33) $\alpha=4, a=1, b=2.5, c_{4}=13$

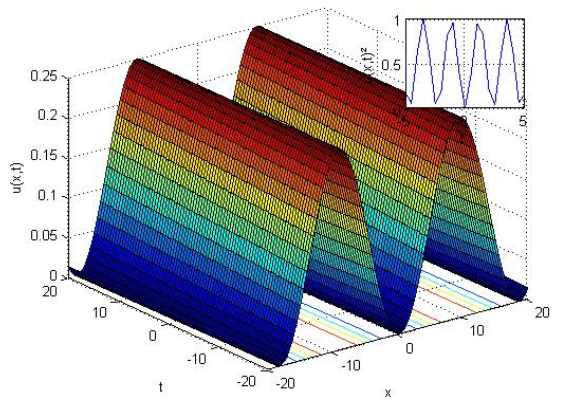

Figure 3: 3D plot of (23) $\alpha=80, \beta=10, a=3, b=2.5$, $c_{3}=0.5, c_{2}=c_{4}=10$

The following are the conserved vectors obtained from the four infinitesimals say $X_{1}, X_{2}, X_{3}$, and $X_{4}$ when $C_{1}=1$ respectively.

$$
\begin{aligned}
& C^{1}=\frac{1}{4}\left(\left(-2+4 b u_{x}\right) u_{x t}+u_{x x x t}\right) \\
& C^{2}=-\frac{1}{4}\left(\left(-2+4 b u_{x}\right) u_{x x}+u_{x x x x}\right) .
\end{aligned}
$$

$$
\begin{aligned}
& C^{1}=\frac{1}{2}(a-2 b) u_{x t}, \\
& C^{2}=-\frac{1}{2}(a-2 b) u_{x x} .
\end{aligned}
$$

Similarly, one can verify and see that $D_{x} C^{1}+D_{t} C^{2}=0$ which is a trivial conservation laws.

$$
\begin{aligned}
& C^{1}=\frac{1}{4}\left(u_{t t}\left(2-2 a u_{x}\right)+\left(4-2 a u_{t}\right) u_{x t}-3 u_{x x t t}\right), \\
& C^{2}=\frac{1}{4}\left(2\left(-1+a u_{x}\right) u_{x t}+2\left(-2+a u_{t}\right) u_{x x}+3 u_{x x x t}\right) .
\end{aligned}
$$

$$
C^{1}=\frac{1}{4}\left\{-8+8 a u_{x}+2 a t u_{t t}\left(-1+a u_{x}\right)\right.
$$

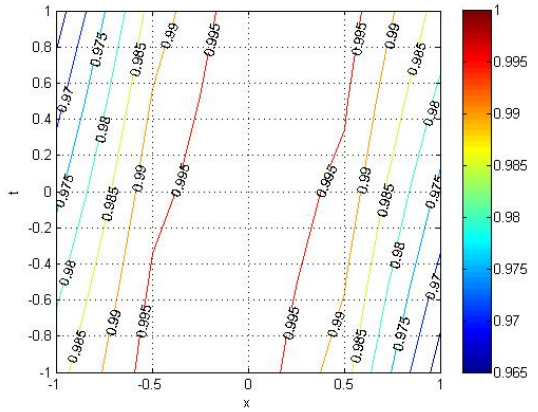

Figure 2: contour plot of (33) $\alpha=80, c_{1}=5, c_{2}=0.5$

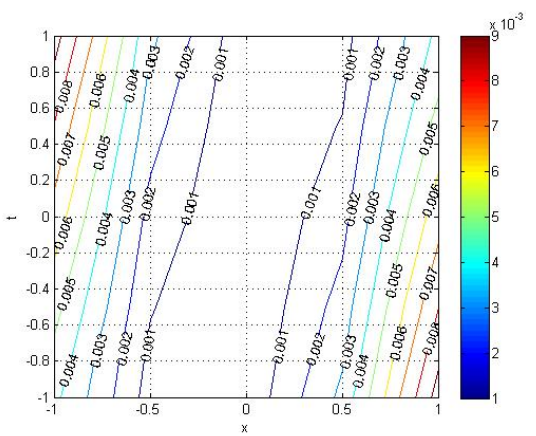

Figure 4: contour plot of (23) $\alpha=80, \beta=10, a=3, b=2.5$, $c_{3}=0.5, c_{2}=c_{4}=10$

$$
\begin{aligned}
& -4 a t u_{x t}+2 a x u_{x t}-8 b x u_{x t} \\
& -2 a^{2} u(x, t) u_{x t}+4 a b u(x, t) u_{x t} \\
& +4 a b x u_{x} u_{x t}+u_{t}\left(8 b-8 a b u_{x}+2 a^{2} t u_{x t}\right) \\
& \left.-a u_{x x t}+3 a t u_{x x t t}+a x u_{x x x t}\right\}
\end{aligned}
$$

$$
\begin{aligned}
C^{2}=- & \frac{1}{4}\left\{4+4 a^{2} u_{x}^{2}-2 a t u_{x t}\right. \\
& -4 a t u_{x x}+2 a x u_{x x}-8 b x u_{x x}-2 a^{2} u(x, t) u_{x x} \\
+ & 4 a b u(x, t) u_{x x}+2 a^{2} t u_{t} u_{x x}+2 a u_{x}\left(-4+a t u_{x t}\right. \\
& \left.\left.+2 b x u_{x x}\right)+4 a u_{x x x}+3 a t u_{x x x t}+a x u_{x x x x}\right\} .
\end{aligned}
$$

\section{Conclusion}

In this study, Lie symmetry analysis, one dimensional optimal system and Cls for GSWW equation were studied. Some reductions and their solutions were reported from the obtained one dimensional optimal system. We presented some figures for some of the obtained exact solutions. The exact solutions include an expression with a Bessel function and an Airy function. We verified the au- 
thenticity of the solution by substitution into the original equation. The GSWW equation is a NSA with the obtained differential substitution, we obtained Cls using the new conservation theorem presented by Ibragmov.

\section{References}

[1] Temme N.M., Special Functions: An Introduction to the Classical Functions of Mathematical Physics, 2011, John Wiley and Sons, DOI: $10.1002 / 9781118032572$

[2] Vallee O., Soares M., Airy Functions and Applications to Physics, 2004, Imperial College Press

[3] Temme N.M., Special Functions: An Introduction to the Classical Functions ofMathematical Physics, 1996, John Wiley and Sons

[4] Emrullah Y., San S., Özkan Y.S. "Nonlinear self ad-jointness, conservation laws and exact solutions of ill-posed Boussinesq equation, Open Physics, 2016, 14(1), 37-43.

[5] San S., Akbulut A., Ünsal Ö, Tascan F. Conservation laws and double reduction of $(2+1)$ dimensional CalogeroBogoyavlenskii-Schiff equation, Math. Meth. Appl. Sci., 2017, 40(5), 1703-1710.

[6] Emrullah Y., San S., A procedure to construct conservation laws of nonlinear evolution equations, Zeitschrift für Naturforschung A, 2016, 71(5), 475-480.

[7] Inc M., Aliyu A.I., Yusuf A., Dark optical, singular solitons and conservation laws to the nonlinear Schrödinger's equation with spatio-temporal dispersion, Mod. Phys. Let. B, 2017, 31(14), 1750163

[8] Tchier F., Yusuf A., Aliyu A.I., Inc M., Soliton solutions and Conservation laws for Lossy Nonlinear Transmission line equation, Superlat. Microstr., 2017,107, 320-336.

[9] Inc M., Aliyu A.I., Yusuf A., Traveling wave solutions and conservation laws of some fifth-order nonlinear equations, Eur. Phys. J. Plus, 2017, 132, 224.

[10] Bokhari A.H., Al Dweik A.Y., Kara A.H., Zaman F.D. A symmetry analysis of some classes of evolutionary nonlinear $(2+1)$ - diffusion equations with variable diffusivity, Nonlinear Dyn., 2010, 62, 127-138.

[11] Krishnan E.V., Triki H., Labidi M., Biswas A., A study of shallow waterwaveswith Gardner's equation, Nonlinear Dyn, 2011, 66, 497-507.

[12] Biswas A., Kara A.H., 1-Soliton solution and conservation laws of the generalized Dullin-Gottwald-Holm equation, Appl. Math. Comp., 2010, 217, 929-932.

[13] Biswas A., Kara A.H., Moraru L., Bokhari A.H., Zaman F.D., Conservation Laws of Coupled Klein-Gordon equation with cubic and power law nonlinearities, The publishing house proceeding of the Romanian Academy, Series A, 2014, 15(2), 123-129.

[14] Ebadi G., Kara A.H., Petkovic M.D., Biswas A., Soliton solutions and conservation laws of the Gilson-Pickering equation, Waves in Random and Complex Media, 2011, 21(2), 378-385.

[15] Morrisa R., Kara A.H., Biswas A., Soliton solution and conservation laws of the Zakharov equation in plasmas with power law nonlinearity, Nonlinear Analysis: Modelling and Control, 2013, 18(2), 153-159.

[16] Razborova P., Kara A.H., Biswas A., Additional conservation laws for Rosenau-KdV-RLW equation with power law nonlinearity by Lie symmetry, Nonlinear Dyn., 2015, 79, 743-748.
[17] Noether E., Invariant variation problem, MathematischPhysikalische Klasse, 1918, 2, 235-257.

[18] Khamitova R., Symmetries and Conservation laws, PhD thesis, 2009, Växjö, Sweden

[19] Ibragimov N.H., A new Conservation laws theorem, J. Math. Anal., 2007, 333(1), 311-328.

[20] Ibragimov N.H., Nonlinear self-adjointness in constructing conservation laws, Archives of ALGA, 2010, 7/4, 1-18.

[21] Ibragimov N.H., Nonlinear self-adjointness in constructing conservation laws, Archives of ALGA, 2011, 44(43), 2011

[22] Ovsyannikov L.V., Group analysis of differential equations, Nuaka, Moscow, 1978, English transl., Ames W.F. (Ed.), 1982, Academic Press, New York.

[23] Ovsyannikov L.V., Group properties of differential equations, 1962 Siberian Branch, USSR Academy of Sciences, Novosibirirsk, (Russian)

[24] Patera J., Winternitz P., Zassenhaus H., Continuous subgroups of the fundamental groups of physics, General method and the Poincare group, J. Math. Phys., 1975, 16, 1597-1614.

[25] Patera J., Sharp R.T., Winternitz P., Zassenhaus H., Invariants of real low dimension Lie algebras, J. Math. Phys., 1976, 17, $986-$ 994.

[26] Galas F., Richter E.W., Exact similarity solutions of ideal MHD equations for plane motions, Phys. D, 1991, 50, 297-307.

[27] Gomez C.A., Salas A., Exact solutions for the generalized shallow water wave equation by the general perspective Ricatti equations method, Math. Phys., 2006, 2006, 50-56.

[28] Inc M., Ergut M., Periodic wave solutions for the generalized shallow water wave equation by the improved Jacobi elliptic function method, Appl. Math. E-Notes, 2005, 5, 89-96.

[29] Elwakil S.A., El-labany S.K., Zahran M.A., Sabry R., Exact travelling wave solutions for the generalized shallow water wave equation, Chaos, Solitons \& Fractals, 2003, 17(1), 121-126.

[30] Bagchi B., Das S., Ganguly A., New exact solutions of a generalized shallow water wave equation, Phys. Scr., 2010, 82(2), 025003

[31] Dimas S., Tsoubelis D. SYM: A new symmetry finding pack- age for Mathematica, In: Ibragimov N.H., Sophocleous C., Damianou P.A., (Eds.), The 10th International Conference in Modern Group Analysis, 2005, 64-70, Nicosia, University of Cyprus

[32] Bilby B.A., Miller K.J., Willis J.R., Fundamentals of Deformation and Fracture, 1985, Cambridge Univ. Press, Cambridge

[33] Knops R.J., Stuart C.A., Quasiconvexity and uniqueness of equilibrium solutions in nonlinear elasticity, Arch. Rat. Mech. Anal., 1984, 86, 234-249.

[34] Harwitt M., Photon orbital angular momentum in astrophysics, Astrophys. J., 2003, 597, 1266-1270.

[35] Elias N.M., Photon orbital angular momentum in astronomy, Astron. Astrophys, 2008, 492(3), 883-922.

[36] Berkhout G.C.G., Beijersbergen M.W., Method for probing the orbital angular momentumof optical vortices in electromagnetic waves from astronomical objects. Phys. Rev. Lett., 2008, 101, 100801

[37] Thidé B., Then H., Sjöholm J., Palmer K., Bergman J., Carozzi T.D., Istomin Y.N., Ibragimov N.H., Khamitova R., Utilization of photon orbital angular momentum in the low-frequency radio domain, Phys. Rev. Lett., 2007, 99, 087701-1-087701-4 